\title{
Stress Monitoring in Conflict Resolution Situations
}

\author{
Davide Carneiro ${ }^{1}$, José Carlos Castillo ${ }^{2}$, Paulo Novais ${ }^{1}$, Antonio Fernández- \\ Caballero $^{2}$, José Neves ${ }^{1}$, María T. López ${ }^{2}$ \\ ${ }^{1}$ Department of Informatics/CCTC \\ University of Minho, Braga, Portugal \\ \{dcarneiro, pjon, jneves\}@di.uminho.pt \\ ${ }^{2}$ Instituto de Investigación en Informática de Albacete (I3A) \\ Universidad de Castilla-La Mancha, Albacete, Spain \\ \{JoseCarlos.Castillo, Antonio.Fdez, Maria.LBonal\}@uclm.es
}

\begin{abstract}
Online Dispute Resolution is steadily growing to become the major alternative to litigation in court. In fact, given the characteristics of current disputes, technology-based conflict resolution may be a quite efficient approach. However, in this shift of paradigm, there are also threats that should be considered. Specifically, in this paper we deal with the problem of the lack of important context information when parties communicate in a virtual setting. In that sense, we propose the addition of a monitoring framework capable of measuring the level of stress of the parties in a non-invasive way. This information will be used by the platform and the mediator throughout the complete conflict resolution process to adapt strategies in real-time, resulting in a context-aware and more efficient approach.
\end{abstract}

Keywords: Online Dispute Resolution, Stress Monitoring, Monitoring Framework

\section{Introduction}

The field of conflict resolution is historically related to the Social Sciences field. However, the advent of technology brought along significant changes in the way that conflicts emerge and are solved [11]. This is especially visible in electronic contracting, in which the soundest example is e-commerce. This has led to the emergence of the so-called Online Dispute Resolution (ODR) [1], encompassing techniques or approaches for conflict resolution taking partly or entirely place in a virtual environment, with a varying autonomy of technology [2].

Nevertheless, ODR also presents some threats [3]. One of the most significant is the important context information that is lost when parties do not communicate 
in the physical presence of each other. Particularly significant is all the information from the body language that we (unconsciously) rely on in our day-to-day interactions. In fact, Mehrabian [4] states that the non-verbal elements of a conversation are particularly important for communicating feelings and attitudes. Thus, the problem is that information is lost in a virtual setting and makes it hard for the intervenient parties to understand the emotional state of each other.

In this paper we describe a context-aware environment to support a conflict resolution platform by providing important information about the state of the parties [5]. Specifically, we focus on the estimation of the level of stress of the intervenient. The aim is to improve the awareness of user's states in current ODR processes, allowing both parties and neutrals to take better and more informed decisions, ultimately increasing the efficiency of the conflict resolution process.

\section{System Overview}

Information about the user's context is provided through a monitoring framework [7], which is customized to perform movement detection from a camera located in front of the user. Considering frame-to-frame movement information, the level of agitation or excitement of the user can be analyzed. From these movements, more complex behaviors can be analyzed to detect stress patterns in the form of activities [10]. Moreover, recent works deal with the detection of emotions by employing visual sensors [9]. Additionally, we are relying on the use of interfaces developed for touch screens empowered with accelerometers that allow capturing information about how the user interacts with the conflict resolution system. Specifically, we are interested in information about the intensity and the accuracy of the touches, basically the users touch patterns. Combining this information, an estimation of the level of stress of a user can be computed and provided to the conflict resolution platform (Figure 1).

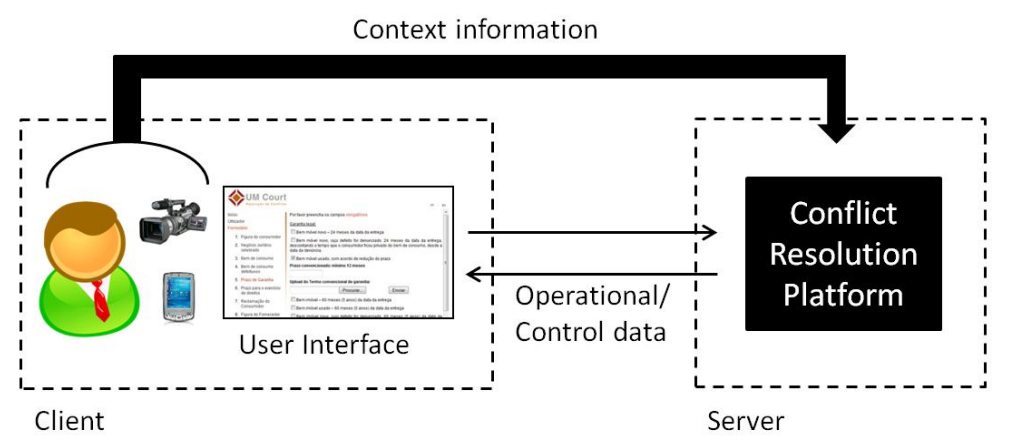

Fig. 1. Overview of the developed system: context information is provided to the conflict resolution platform, which interpret the user's state. 
Information about stress is very important in a conflict resolution scenario, as it will allow the mediator or the platform itself to adapt to significant changes in the user state. Concrete adaptations include temporarily interrupting the direct contact between the parties when they become too stressed, making a pause in the process, or even proposing an outcome that satisfies more precisely the expectations of the more stressed party, in an attempt to calm him/her down and keep him/her focused on the process. In the following sections we describe in detail the approach used, the experiments performed and the results achieved.

\section{Monitoring the User Stress from Visual Information}

To detect the excitement degree of a user, the framework recently presented in [7] has been particularized to monitor the movements of a user seated in front of a camera while interacting with the ODR touch interface (Figure 2). After data acquisition, a timestamp is associated to the images to enable them to be matched with the touch information. The amount of movement is calculated as the amount of pixels that have changed their RGB color value over a given threshold between two consecutive images. Although the algorithm is deeply described in [8], including an implementation focused on the infrared imaging technology, the main steps are highlighted next. Firstly the subtraction, $I_{S}$, of the current image, $I_{t}$, and the previous one, $I_{t-1}$, is calculated as follows:

$$
I_{S}(i, j)=\left|I_{t}(i, j)-I_{t-1}(i, j)\right|
$$

Afterwards, it is necessary to determine which pixels in $I_{S}$ have higher changes, just in order to discard noise. That is why a binarization process is carried out. Thus, pixels over the established threshold, $\tau$, are set to 1 and those pixels below $\tau$ are set to 0 as described next, obtaining the binarized image, $B$.

$$
B(i, j)= \begin{cases}0, & I_{S}(i, j)<\tau \\ 1, & I_{S}(i, j) \geq \tau\end{cases}
$$

After the binarization, some filters are applied to $B$ in order to reduce the noise impact and to enhance the really relevant movements. For this purpose, some opening and closing morphological operations are applied, obtaining $B^{F}$. Finally, the amount of movement is calculated as the number of non-zero pixels on $B^{F}$ :

$$
M=\sum_{j=0}^{\text {height }} \sum_{i=0}^{\text {width }} B^{F}(i, j)
$$


a)

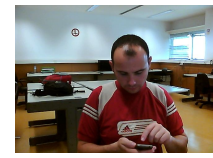

b)

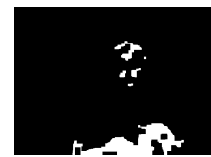

c)

$\mathrm{M}=\mathbf{1 8 1 5 4}$
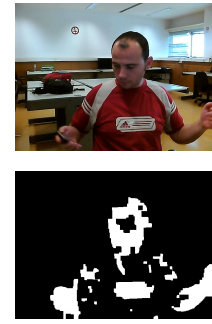

$M=40985$
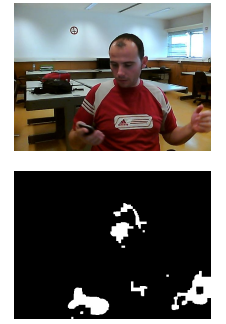

$M=18554$
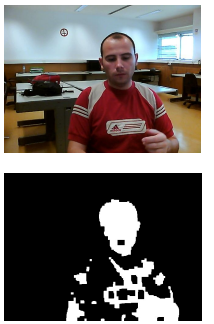

$M=42696$
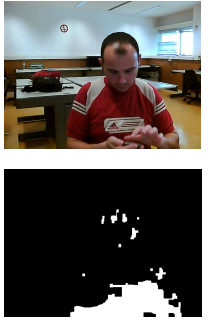

$M=26618$

Fig. 2. Results of movement detection of a user whilst interacting with the conflict resolution platform. Row a) shows input images, row b) shows binarized and filtered movement and row c) shows the amount of movement detected.

\section{Collecting Information from User Interaction}

The effects of stress are generally visible in almost all of our behaviors. In that sense, it is possible to establish a relation between the effects observed and the level of stress. Specifically, we are interested in how stress affects the user's touch patterns while using the interface of the application. In that sense, the proposed system constantly collects information about the user's touch intensity and accuracy. The approach is based on the notion that a higher level of stress results in a higher touch pressure and a lower accuracy and vice versa. The information collected is sent to the UMCourt conflict resolution platform [6], which interprets it and uses it as an additional input for managing the conflict resolution process.

The data is always analyzed taking into consideration the context in which it was acquired. In that sense, data generated from the management (e.g. managing personal information, accessing past cases) and login interfaces are used to calibrate the system and to establish a baseline for the level of stress. This comprises the training phase. On the other hand, data generated from the current conflict resolution interfaces are used to assess the level of stress of the user. It constitutes the operational phase. This is important as two individuals are in no case affected by the stress in the same way. In order to compute a measure of accuracy, touch interfaces have two areas: active and passive (see Figure 3). Active areas are defined by active controls used by the user to perform his/her tasks. Passive areas have no controls and there is no reason why a user should touch them. This allows measuring the accuracy of the touch by comparing the total number of touches versus the touches in active or passive areas.

To measure the intensity of the touch, the whole touch action is analyzed, since the finger of the user firstly touches the screen and then he/she releases it. In fact, differences between a touch performed by a stressed user and by a user in a normal state are easily visible (as shown in Figure 4). In this work, it can be concluded that, in general, the touch pattern of a stressed user has a longer duration and 
starts with an increasing pressure up to a maximum reached and then it decreases until the release of the finger. On the other hand, the touch pattern of a user in a normal state usually starts in or near a maximum value of pressure, which then decreases until the finger is released.
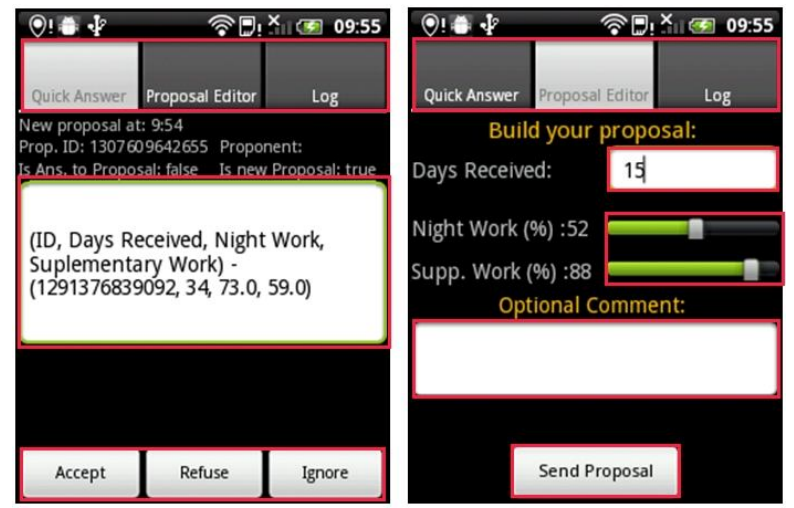

Fig. 3. A detail of the touch user interfaces used in the experiment: colored rectangles define active areas while the rest are passive areas.

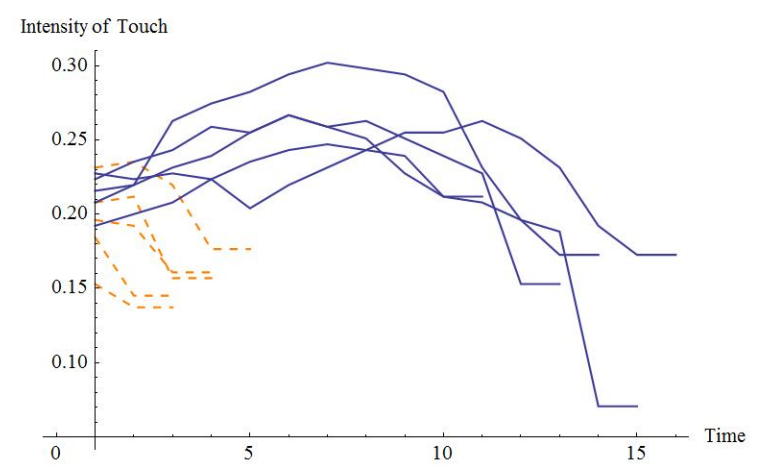

Fig. 4. Plot of the variation of the touch intensity over time from touch events generated by a user in a normal state (dashed orange line) and in a stressed state (blue line).

\section{Experiments and Results}

To perform the experiments detailed in this paper, we place the users of the conflict resolution platform in front of video cameras, while they interact with the touch interfaces. The data collected from the touch interfaces consist of a list of touch events. Each entry includes the timestamp in which it occurs, the maximum, minimum and mean value of the touch intensity as well as the target of the touch, i.e. passive or active. As stated before, the data collected from the camera consists 
of a sequence of images, captured from a frontal view of the user. The images are associated to a timestamp to allow the correspondence with the touch events. As an output, the amount of movement from the color input images is obtained.

Given that the visual system generates much more information than the touch interfaces, a recurrent task is to group parts of the information from the camera. The first analysis of the information starts by grouping all the information from the camera generated between two consecutive touch events $T_{t-1}$ and $T_{t}$. This allows to compare the intensity of a given touch event $T$ and the amount of movement in the time interval defined by $] t-1, t]$. For this data, the Pearson's correlation coefficient between the intensity of the touch and the sum of the ratio of movement for the defined time interval returns values around 0.5 .

However, this approach may be misleading as it considers the whole interval between touch events and there are cases in which users move away their focus of attention from the application. In that sense, alternative ways of handling the information are introduced. Firstly, we start analyzing a reduced time interval $\Delta$ immediately before each touch event, under the conviction that immediately before interacting with the interface, the user's attention is directed towards the conflict resolution. Thus, for each touch event $T$ in time $t$, we analyze the information from the video camera in the interval defined by $\llbracket t-\Delta, t \rrbracket$. This consists in computing the sum of the movement in each interval and comparing it with the intensity of the touch. The experiment shows a Pearson's correlation of around 0.68 .

We also analyze a concrete interval, which is the most significant in terms of stress in a conflict resolution system: an interval after receiving a new proposal. In fact, when parties receive and read a new proposal, they tend to express in a more explicit way their frustration or their contentment. In that sense, when considering only intervals of data starting in each new proposal and lasting for an interval $\Delta$, the highest value of correlation between intensity and amount of movement is visible, namely around 0.74 . Figure 5 shows a plot of data extracted from a negotiation session where 10 solutions are proposed by the platform. The correlation of the data is visible.

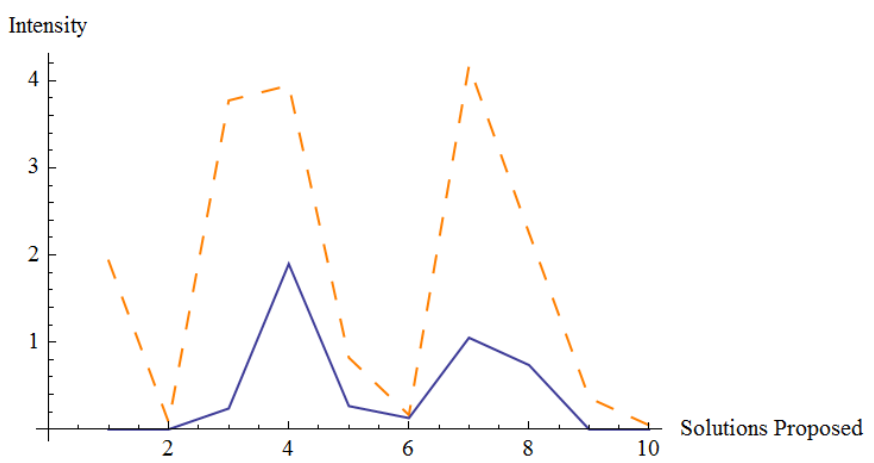

Fig. 5. Plot of data concerning the sum of the intensity of the touches (dashed plot) and the mean rate of movement after the platform proposes a solution. 
Two other representations of the collected data allow seeing this relation between increased amount of movement and intensity of the touch in stressed users. In the first one (see Figure 6a)), the intensity of the touch versus the sum of the movement in the period after the last touch and before the current one is analyzed. It is possible to conclude that, in a general way, the touches with higher intensity are preceded by higher movement rates. The second representation of the data depicts the movement immediately before touch events (see Figure 6 b)). Here, it is also possible to see that touch events with smaller intensity are associated to less movement, and vice versa.

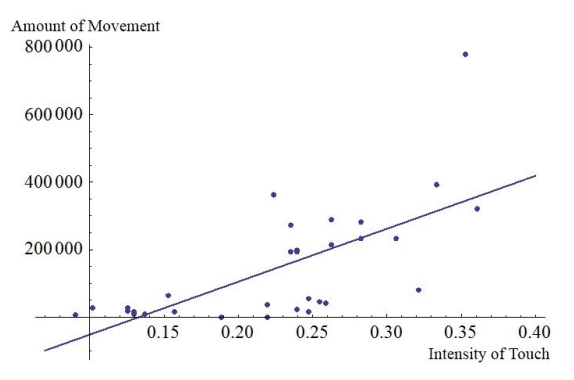

a)

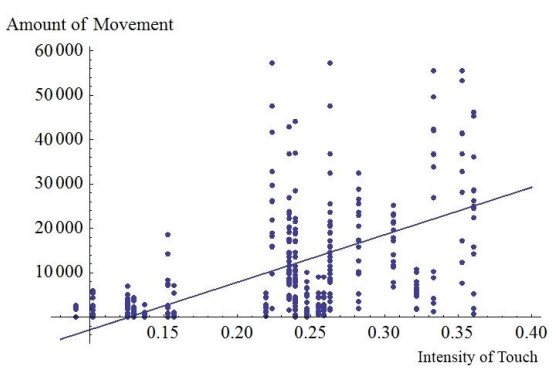

b)

Fig. 6. Two different representations of the data, highlighting the relation between the amount of movement and the intensity of the touch.

\section{Conclusions and Future Work}

After performing the experiments described in this paper, we are able to conclude that effectively there is a relation between increased user movement and touch intensity and accuracy. Even though, the amount of movement is a first step to the visual detection of the user stress that can be improved by adding more complex techniques, such as methods based in pattern recognition to detect specific movements of the user. Nevertheless, user movement has been proved to be a good stress indicator in combination to other sensors information, such as the touch. Moreover, it can also be concluded that the relation movement-touch is more emphasize in moments of more stress, e.g. after parties receive disadvantageous proposals. In that sense, we can assume that there is a relation between higher levels of stress and higher touch intensities and movements. The approach can thus be an effective and non-invasive way of providing important context information to the conflict resolution platform and to the mediator. By taking in account this information it is possible to build context aware platforms capable of adapting in realtime to the state of the users, aiming for an increased success rate.

This work goes on by including new sources of information that allow a more accurate classification of the level of stress. Specifically, we are considering including information from accelerometers available in handheld devices used for 
user interaction. This will help in finding patterns associated to stress. We are also considering the use of additional technologies, namely electroencephalograms. Moreover, regarding the information provided by the monitoring framework, the current work focuses on the identification of human gestures to detect patterns or activities. Also, the identification of simple emotions will be added to extend the user mood detection to a wider range of human feelings.

Acknowledgments. The work described in this paper is included in TIARAC Telematics and Artificial Intelligence in Alternative Conflict Resolution Project (PTDC/JUR/71354/2006), which is a research project supported by FCT (Science \& Technology Foundation), Portugal. The work of Davide Carneiro is supported by a doctoral grant by FCT (SFRH/BD/64890/2009). This work is also partially supported by the Spanish Ministerio de Ciencia e Innovación under project TIN2010-20845-C03-01 and by the Spanish Junta de Comunidades de Castilla-La Mancha under projects PII2I09-0069-0994 and PEII09-0054-9581.

\section{References}

1. Katsch, E., Rifkin, J.: Online dispute resolution - resolving conflicts in cyberspace. Jossey-Bass Wiley Company, San Francisco. (2001)

2. Peruginelli, G., Chiti, G.: Artificial Intelligence in alternative dispute resolution. Proceedings of the Workshop on the law of electronic agents - LEA. (2002)

3. Larson, D.: Technology Mediated Dispute Resolution. Proceeding of the 2007 conference on Legal Knowledge and Information Systems: JURIX 2007: The Twentieth Annual Conference. IOS Press Amsterdam, The Netherlands (2007)

4. Mehrabian, A.: Silent Messages - A Wealth of Information about Nonverbal Communication. Personality \& Emotion Tests \& Software. Los Angeles (2009)

5. Carneiro, D., Novais, P., Neves, J.: Toward Seamless Environments for Dispute Prevention and Resolution, International Symposium on Ambient Intelligence, Advances in Intelligent and Soft Computing, Springer, Volume 92/2011, 25-32, DOI: 10.1007/978-3-642-19937-0_4 (2011)

6. Carneiro, D., Novais, P., Neves, J.: Towards Domain-Independent Conflict Resolution Tools, The 2011 IEEE/WIC/ACM International Conference on Intelligent Agent Technology (IAT 2011) (2011)

7. Castillo, J. C., Rivas Casado, A., Fernández-Caballero, A., López, M. T., MartínezTomás, R.: A Multisensory Monitoring and Interpretation Framework Based on the Model-View-Controller Paradigm. IWINAC (1): 441-450 (2011)

8. Fernández-Caballero, A., Castillo, J. C., Martínez-Cantos, J. and Martínez-Tomás, R.: Optical flow or image subtraction in human detection from infrared camera on mobile robot. Robotics and Autonomous Systems, 58 (12). Elsevier Science. ISSN 09218890 (2010)

9. Bee Theng, L., Portable real time emotion detection system for the disabled. Expert Systems with Applications, 37(9): 6561- 6566, j.eswa.2010.02.130 (2010)

10. Fernández-Caballero, A., Castillo, J. C., Rodríguez-Sánchez, J. M.: A Proposal for Local and Global Human Activities Identification. AMDO, 78-87 (2010)

11. Katsh, E., Rifkin, J., Gaitenby, A.: E-Commerce, E-Disputes, and E-Dispute Resolution: In the Shadow of eBay Law. Ohio State Journal on Dispute Resolution, 15, 705 (1999) 